\title{
Spin relaxation in CdTe quantum dots
}

\author{
Ye Chen, Tsuyoshi Okuno, and Yasuaki Masumoto \\ Institute of Physics and Venture Business Laboratory, University of Tsukuba, Tsukuba, Ibaraki 305-8571, Japan \\ Yoshikazu Terai,* Shinji Kuroda, and Koki Takita \\ Institute of Material Science, University of Tsukuba, Tsukuba, Ibaraki 305-8573, Japan \\ (Received 28 April 2004; revised manuscript received 28 September 2004; published 25 January 2005)
}

\begin{abstract}
We have measured photoluminescence (PL) spectra and time-resolved PL in CdTe quantum dots under the longitudinal magnetic field up to $10 \mathrm{~T}$. Circular polarization of PL increases with increasing magnetic field, while its linear polarization remains zero under linearly polarized excitation. This behavior cannot be explained by the anisotropic exchange interaction of excitons. Time-resolved PL measurements clarified that this behavior is caused by the suppression of spin relaxation induced by the longitudinal magnetic field. We believe that this behavior is related to the hyperfine interaction of electron spin with magnetic momenta of lattice nuclei.
\end{abstract}

DOI: 10.1103/PhysRevB.71.033314

PACS number(s): 78.66.Hf, 78.67.Hc, 78.20.Ls, 78.47.+p

The spin state of electrons in quantum dots (QDs) is considered as one of the most promising candidates for the implementation of spintronic and quantum information technologies. ${ }^{1}$ A key challenge is to obtain long relaxation time of electron spin. The carrier spin-flip mechanisms, including exchange interaction, mixing between the conduction and valence band states through spin-orbit coupling, and spin splitting of the conduction band due to the lack of inversion symmetry, ${ }^{2,3}$ have been studied in bulk and twodimensional systems. In QDs, the discrete energy levels and the corresponding lack of energy dispersion lead to a predicted modification of the spin relaxation dynamics. In general, the D'yakonov and Perel' mechanism does not work in zero dimension because this spin-flip mechanism relies on translation invariance, which is already broken in QDs. ${ }^{4}$ The electron spin relaxation mechanism connected with the spinorbit interaction of carriers is strongly suppressed for localized carriers in QDs. ${ }^{5}$ The absolute lack of energy states between QD energy levels is expected to inhibit not only the elastic processes of spin relaxation but also the inelastic ones such as phonon scattering. As a result, electron spin relaxation via interaction with nuclei becomes relatively important for localized electrons. Recently, Merkulov et al. ${ }^{6}$ pointed out that the hyperfine interacting with nuclei may become the dominant mechanism of electron spin relaxation in quantum dots at low temperature. Although the hyperfine interaction has been demonstrated to induce the additional Zeeman splitting or polarization of electrons and exciton in QDs, ${ }^{7,8}$ no direct experimental evidence has proved the importance of hyperfine interaction with nuclei responsible for spin relaxation in QDs up to now.

In this report, we investigated the spin relaxation mechanisms of carriers in CdTe QDs. We measured the magnetophotoluminescence (PL) spectra and time-resolved PL under the magnetic field with a Faraday configuration. An unusual magnetic field dependence of circular polarization was observed in steady-state PL spectra, which cannot be explained in the framework of anisotropic exchange interaction of excitons confined in an elongated QD. Time-resolved PL decay proved that the suppression of spin relaxation by the longitudinal magnetic field is the origin of this behavior. We be- lieve that the spin relaxation of electrons localized in QDs is caused mainly by hyperfine interaction of electron spin with magnetic momenta of lattice nuclei. The hyperfine field is suppressed by the external longitudinal magnetic field.

The CdTe self-assembled QD was grown by molecularbeam epitaxy on a relatively thick $(0.6-1.0 \mu \mathrm{m}) \mathrm{ZnTe}$ buffer layer formed on a GaAs (100) substrate. ${ }^{9}$ The sample was not doped intentionally. Atomic force images show that the average size of CdTe QDs was $\sim 20 \mathrm{~nm}$ in diameter and $\sim 2.7 \mathrm{~nm}$ in height. The area density of the dots was $8 \times 10^{10} \mathrm{~cm}^{-2}$. The PL and time-resolved PL measurements were performed in a magnetic field up to $10 \mathrm{~T}$ and at $10 \mathrm{~K}$ in a Faraday configuration. An optical parametric amplifier of a Ti:sapphire laser was used as an excitation source with a photon energy of $2.217 \mathrm{eV}$, corresponding to a quasiresonance excitation of CdTe QDs. Circular and linear polarizations were obtained by using quartz wave plates and linear polarizers, respectively.

The representative PL spectra of our sample are shown in Fig. 1(a). In these measurements, the luminescence was collected under co-circular (the incident and emitted light with the same circular polarization) and cross-circular geometries (the incident and emitted light with counter-circular polarization). In order to study the intrinsic spin dynamics, the PL spectra were obtained by selectively exciting the sample with photon energy $2.217 \mathrm{eV}$ slightly above the PL band of the QDs. The PL bands of the QDs have a smooth profile with two sharp features, $\mathrm{LO}_{1}$ and $\mathrm{LO}_{2}$ (24.5 and $19.5 \mathrm{meV}$, respectively), which are caused by fast relaxation of hot carriers with emission of LO phonons of ZnTe and CdTe.

At zero field, the PL band shows very weak circular polarization $(7 \%)$. With increasing magnetic field the circular polarization of PL band increases. When the direction of magnetic field was reversed, the same results were obtained. We further measured the circularly polarized PL spectra under linearly polarized excitations at $4 \mathrm{~T}$ in Fig. 1(b). It confirms that no circular polarization was observed under linearly polarized excitation. Therefore, the circular polarization is not related to the thermalization between Zeeman split levels.

Figure 2 shows the circular polarization of the emission 


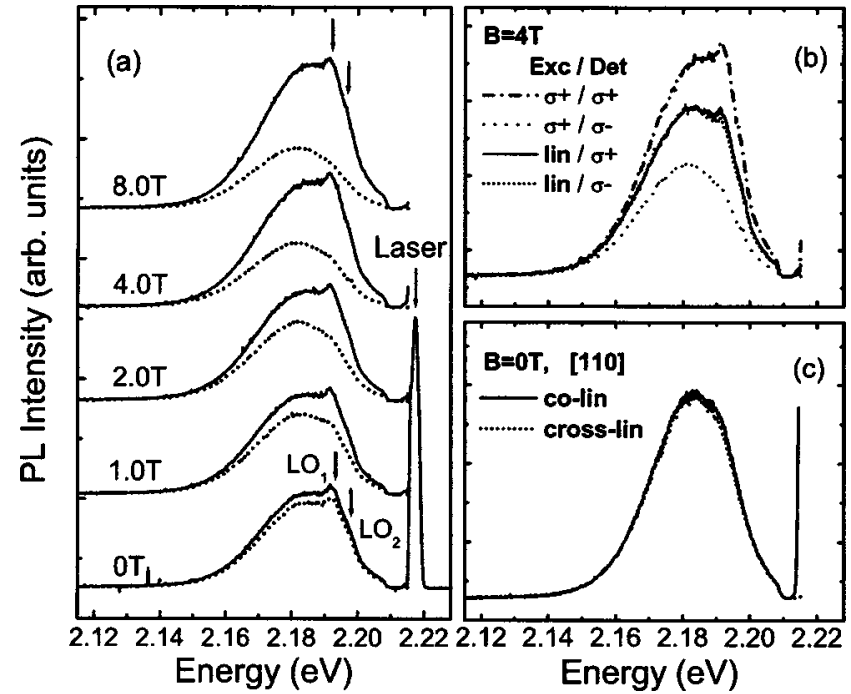

FIG. 1. Circularly polarized PL spectra (a) in co-circular (solid lines) and cross-circular (dotted lines) geometries, at various magnetic fields. (b) Circularly polarized PL spectra in four geometries at $4 \mathrm{~T}$. Linearly polarized excitation gives the same intensity of rightand left-circularly polarized PL (lin $/ \sigma^{+}$and $\operatorname{lin} / \sigma^{-}$, respectively). (c) Linearly polarized PL spectra in co- and cross-linear geometries along [110] direction.

peak as a function of magnetic field. The circular polarization is defined by $P=\left(I^{+}-I^{-}\right) /\left(I^{+}+I^{-}\right)$, where $I^{+}$and $I^{-}$are the PL intensities under the co-circular and cross-circular geometries. It shows that the circular polarization increases with increasing the magnetic field and saturates at the high magnetic field above $6 \mathrm{~T}$. The circular polarization of phonon replica $\mathrm{LO}_{1}$ was also presented in the figure. Figure 1(a) shows that the phonon replica is not polarized at zero field, while it becomes strongly polarized in co-circular geometry with applying magnetic field.

This behavior is quite similar to the effect induced by the anisotropic exchange interaction of excitons. As we know,

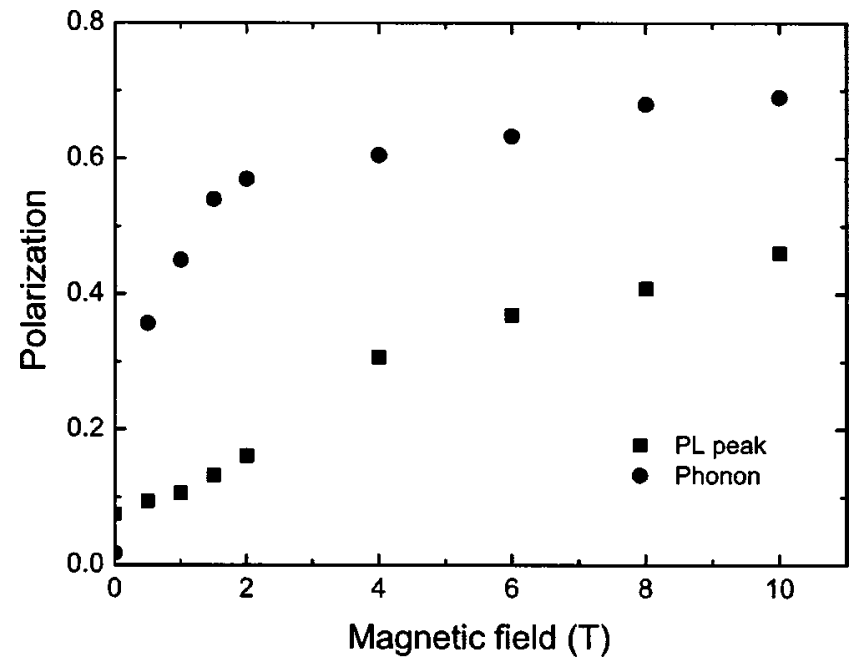

FIG. 2. Magnetic field dependence of the circular polarization of QDs emission peak and phonon resonance $\mathrm{LO}_{1}$ in steady-state PL spectra. the ground state of the e1-hh1 $(1 s)$ heavy-hole exciton in a zinc-blende quantum well is fourfold degenerate with the angular-momentum projection $M=s+j= \pm 1, \pm 2$ on the growth axis $z / /[001]$ of the structure. The exchange interaction splits this state into a radiation doublet $| \pm 1\rangle$ and two closely lying optically inactive singlets, which are a superposition of the $| \pm 2\rangle$ states. In some QD structures, the symmetry is lowered and the exchange interaction is thus no longer isotropic. ${ }^{10,11}$ The anisotropic exchange interaction splits the $| \pm 1\rangle$ radiative doublet into two eigenstates labeled $|X\rangle=(|1\rangle+|-1\rangle) /(\sqrt{2})$ and $|Y\rangle=(|1\rangle-|-1\rangle) /(i \sqrt{2})$, linearly polarized along the [110] and [110] directions, respectively. This anisotropic exchange splitting may originate from QD elongation and interface optical anisotropy. ${ }^{12,13}$

When the magnetic field was applied, and if the Zeeman splitting $\hbar \Omega_{z}=g \mu_{B} \mathrm{~B}$ is much larger than the exchange energy $\hbar \omega$, the QD exciton eigenstates are no longer the $|X\rangle$ and $|Y\rangle$ linearly polarized states, but the $|+1\rangle$ and $|-1\rangle$ circular ones. We thus expect to observe circularly polarized PL under $\sigma$-polarized excitation. This circular polarization was indeed observed by several groups in various nanostructure systems. ${ }^{10-14}$ In our case, we did not observe any linear polarization at zero field under linearly polarized excitation along [110] and [110] directions, as shown in Fig. 1(c). At higher field $B=4 \mathrm{~T}$, the PL spectra under linearly polarized excitation in Fig. 1(b) did not show circular polarization, which contradicts this model. We also checked the linearly polarized time-resolved PL under linearly polarized excitation along [110] and [1 $\overline{1} 0]$ directions, and did not observe any polarization in the temporal profile. All these experimental results indicate that anisotropic exciton fine structure is not responsible for our results. Although the anisotropic exchange energy splitting was observed in elongated QDs, its absence is not strange in the isotropic QDs. Surrounding mixed crystal $\mathrm{Zn}_{x} \mathrm{Cd}_{1-x} \mathrm{Te}$ (Ref. 15) might induce isotropic inner core CdTe QDs.

Another possible explanation of the experimental results is that the circular polarization originates from the suppression of spin relaxation by the longitudinal magnetic field. Our results indicate that the spin relaxation time is very fast at zero field, and the spin orientation is practically destroyed during the electron lifetime. If we assume that the spin relaxation is suppressed by the longitudinal magnetic field, then the circular polarization of steady-state PL band increases with increasing magnetic field. We can use a common formula to estimate the spin relaxation time from the steady-state PL measurements:

$$
P=\frac{P_{0}}{1+2 \tau_{r} / \tau_{s}} .
$$

Here, $P_{0}$ is the initial spin polarization, and $\tau_{r}$ and $\tau_{s}$ are the lifetime and spin relaxation time of carriers, respectively. The lifetime $\tau_{r}$ and the initial spin polarization $P_{0}$ use the values obtained from the time-resolved PL measurements, which will be discussed later. (The calculated spin relaxation rates from the steady-state PL polarization degree will be shown in Fig. 4.) 


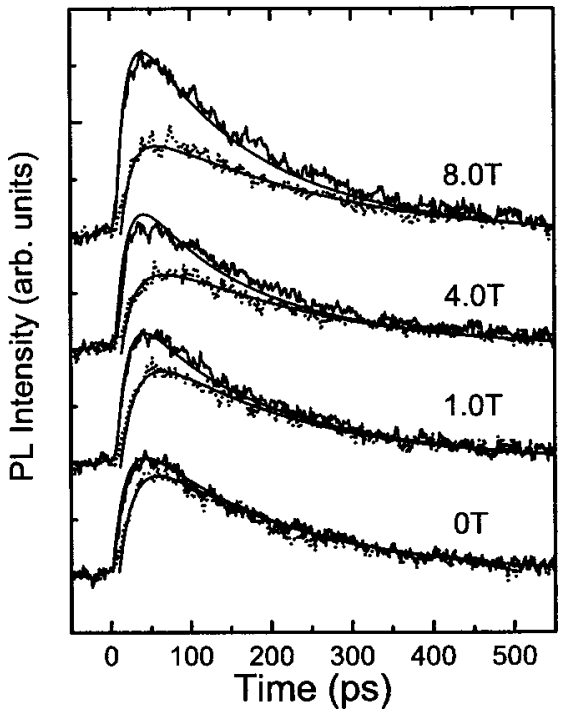

FIG. 3. Time dependence of the circularly polarized PL for cocircular (solid lines) and cross-circular (dotted lines) geometries at various magnetic fields $(0-8 \mathrm{~T})$ at $10 \mathrm{~K}$. Smooth curves are fittings by a combination of a rise function and two exponential decay functions, whose decay times are related to $\tau_{r}$ and $\tau_{s}$.

The protuberant phonon structure observed in the cw luminescence band of QDs is formed by the competition between the phonon-mediated relaxation and nonradiative loss. ${ }^{16}$ Further, in case of the circularly polarized luminescence, the competition between the phonon-mediated relaxation and spin relaxation is thought to determine the phonon structures in the cross-circularly polarized luminescence spectra. In this model, the assumption of suppression of spin relaxation can be used to explain the circular polarization of phonon structures simply. As is seen in Fig. 1(a), LO phonon structures in the cross-circular polarization geometry decrease with the increase of the magnetic field, while those in the co-circular polarization geometry does not change. This feature is explained by the assumption that spin flip time becomes longer than the time for the relaxation mediated by the LO phonon plus acoustic phonons with the increase of the magnetic field.

The time-resolved PL measurements confirmed the suppression of spin relaxation by external magnetic field. The temporal profiles of circularly polarized PL signal under the co-circular and cross-circular geometries at various magnetic fields are shown in Fig. 3. We can see clearly that the spin relaxation time becomes longer with increasing magnetic field. In order to investigate the spin dynamics and recombination process, we calculated the sum $\left(I^{+}+I^{-}\right)$and the difference $\left(I^{+}-I^{-}\right)$of the circularly polarized luminescence, where $I$ is the intensity of circularly polarized PL. The sum $\left(I^{+}+I^{-}\right)$is proportional to $\exp \left(-t / \tau_{r}\right)$, where $\tau_{r}$ is the lifetime. We obtained the lifetime at the maximum position of the PL band, $\tau_{r}=190 \mathrm{ps}$, which is independent of magnetic field. The time-dependent degree of circular polarization $P(t)$ is defined by $P(t)=\left(I^{+}-I^{-}\right) /\left(I^{+}+I^{-}\right) . P(t)$ is proportional to $\exp \left(-2 t / \tau_{s}\right),{ }^{17}$ where $\tau_{s}$ is the spin relaxation time. The spin relaxation time can be estimated from the decay of $P(t)$. The obtained spin relaxation rates, $1 / \tau_{s}$, are shown as a function

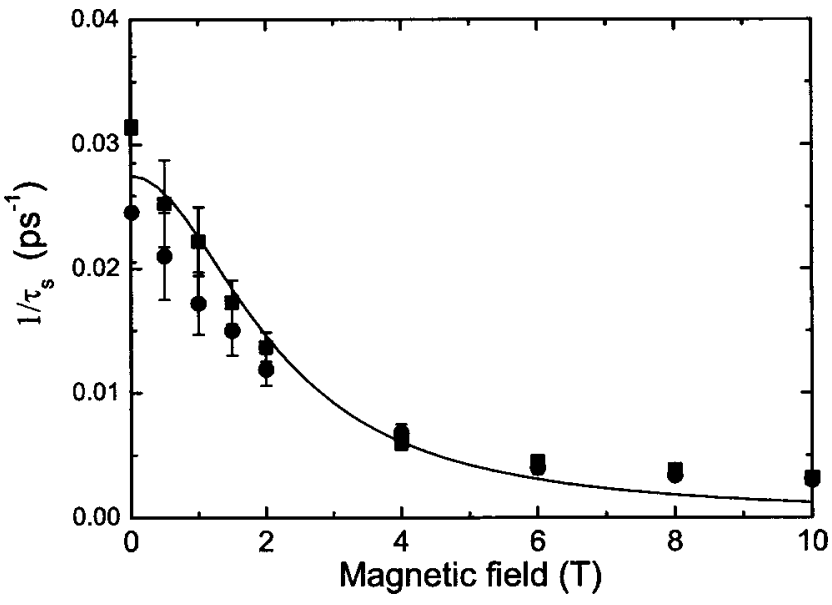

FIG. 4. Magnetic field dependence of the spin relaxation rate. The experimental values from time-resolved PL (circles) and the estimated values from steady-state PL spectra (squares) are shown together for comparison. The solid line is the theoretical fitting according to Eq. (2).

of magnetic field in Fig. 4 by circles. We can see that the measured spin relaxation rates are consistent with the estimated ones from steady-state PL spectra (squares). The discrepancy at low field may come from the fluctuation of laser power, or the difference of detected energies in PL and timeresolved PL measurements. The time-resolved PL measurements proved further that the unusual magnetic field dependence of circular polarization observed in steady-state PL measurements originates from the suppression of spin relaxation by the longitudinal magnetic field.

The spin relaxation rate is not affected remarkably by the magnetic field in the framework of anisotropic exchange interaction of exciton, as was reported by Paillard et al. ${ }^{14} \mathrm{How}-$ ever, we observed that the spin relaxation rate decreases remarkably with the increase of the longitudinal magnetic field. Therefore, the model of the anisotropic exchange interaction of excitons cannot explain our results. We need to consider other possibilities. A suppression of spin relaxation by the external magnetic field has been reported for the case of spin relaxation of electrons localized at donors in bulk crystal. ${ }^{18,19}$ These authors have found that spin relaxation of electrons localized in potential wells is caused mainly by hyperfine interaction of electron spins with magnetic momenta of lattice nuclei, the hyperfine magnetic field being randomly changed due to the hopping migration of electrons over localized states in the crystal. This mechanism should work for localized electrons in QDs. In a strong magnetic field, the nuclear hyperfine fields only perturb the precession frequency of the electron spin about the external magnetic field. As a result, the spin component parallel to $B$ is conserved, while the transverse spin component precesses with Larmor frequency. The dependence of the electron spin relaxation time $\tau_{s}$ on the longitudinal magnetic field can be described by the expression

$$
\frac{1}{\tau_{s}}=\frac{1}{\tau_{s}(0)\left(1+\Omega^{2} \tau_{c}^{2}\right)}
$$

Here, $\tau_{s}(0)$ is the spin relaxation time under the zero magnetic field, $\Omega=g_{e} \mu_{\mathrm{B}} B / \hbar$ is the precession frequency of the 
electron spin in the field $B$, and $\tau_{c}$ is a characteristic time of the order of correlation time of the fluctuating magnetic field responsible for the spin relaxation. Here we used $\tau_{c}$ as a fitting parameter. If the nuclear field $B_{N}$ is present, the quantity in Eq. (2) is defined by the total field acting on the electron spin. By this formula, we can simulate the magnetic field dependence of spin relaxation time, which is in good agreement with the experimental data.

Merkulov et al. studied theoretically electron spin relaxation in InAs QDs and CdSe QDs via interaction with nuclear spins, and they proposed the hyperfine interaction is the dominant mechanism of carriers in these QDs. ${ }^{6}$ They obtained the spin dephasing time on the order of 50-100 ps for InAs QDs, which is close to our data. Correspondingly, we think that the fast relaxation in CdTe QDs is due mainly to the hyperfine interaction with nuclei spin at zero field. The spin relaxation is suppressed by the external magnetic field. However, the hyperfine interaction constants in CdTe are not experimentally determined. Reliable quantitative calculation is difficult to obtain. In this material only a fraction of the nuclei (25\% of the Cd ions) have magnetic moments, and they have spin $I=1 / 2$. As a result, the electron spin interaction with the nuclei in these QDs is weak. Although Merku- lov et al. calculated the hyperfine interaction in nanocrystal CdSe QDs, the obtained spin dephasing time is around $1 \mathrm{~ns}$, and longer than our data. Further theoretical and experimental work is needed to clarify the spin relaxation time for various materials.

In conclusion, we have measured the PL spectra and timeresolved PL in the CdTe/ZnTe QDs under a longitudinal magnetic field up to $10 \mathrm{~T}$ and at $10 \mathrm{~K}$. An unusual magnetic field dependence of circular polarization was observed in steady-state PL spectra. However, we did not observe any linear polarization at zero field. This cannot be explained by the anisotropic fine structures of excitons. Time-resolved PL confirmed that electron spin relaxation is suppressed by the longitudinal magnetic field. We attribute it to the effect of nuclear field. We believe that the spin relaxation is determined mainly by the hyperfine interaction of electrons with nuclei. The Larmor precession of electron spin about the strong external magnetic field can suppress the precession about the internal hyperfine field, thus preserving the initial orientation of electron spins. We expect our experiments can help to understand the spin relaxation processes and to extend the relaxation time in CdTe QDs.
*Present address: Department of Materials Science, Osaka Prefecture University, Sakai, Japan.

${ }^{1}$ S. A. Wolf, D. D. Awschalom, R. A. Buhrman, J. M. Daughton, S. von Molnar, M. L. Roukes, A. Y. Chtchelkannova, and D. M. Treger, Science 294, 1488 (2001).

${ }^{2}$ Optical Orientation, in Modern Problems in Condensed Matter Sciences Vol. 8, edited by F. Meier and B. Zakharchenya (NorthHolland, Amsterdam, 1984).

${ }^{3}$ M. Z. Maialle, E. A. de Andrada e Silva, and L. J. Sham, Phys. Rev. B 47, 15776 (1993).

${ }^{4}$ M. I. D'yakonov and V. I. Perel', Zh. Eksp. Teor. Fiz. 60, 1954 (1971) [Sov. Phys. JETP 33, 1053 (1971)].

${ }^{5}$ A. V. Khaetskii and Y. V. Nazarov, Phys. Rev. B 61, 12639 (2000).

${ }^{6}$ I. A. Merkulov, Al. L. Efros, and M. Rosen, Phys. Rev. B 65, 205309 (2002).

${ }^{7}$ D. Gammon, Al. L. Efros, T. A. Kennedy, M. Rosen, D. S. Katzer, D. Park, S. W. Brown, V. L. Korenev, and I. A. Merkulov, Phys. Rev. Lett. 86, 5176 (2001).

${ }^{8}$ R. I. Dzhioev, B. P. Zakharchenya, V. L. Korenev, and M. V. Lazarev, Phys. Solid State 41, 2014 (1999) [Fiz. Tverd. Tela (Leningrad) 41, 2193 (1999)].

${ }^{9}$ Y. Terai, S. Kuroda, K. Takita, T. Okuno, and Y. Masumoto, Appl.
Phys. Lett. 73, 3757 (1998).

${ }^{10}$ D. Gammon, E. S. Snow, B. V. Shanabrook, D. S. Katzer, and D. Park, Science 273, 87 (1996).

${ }^{11}$ E. L. Ivchenko, Pure Appl. Chem. 67, 463 (1995); R. I. Dzhioev et al., Phys. Solid State 40, 790 (1998).

${ }^{12} \mathrm{~S}$. Hameau, Y. Guldner, O. Verzelen, R. Ferreira, G. Bastard, J. Zeman, A. Lemaître, and J. M. Gérard, Phys. Rev. Lett. 83, 4152 (1999).

${ }^{13}$ R. I. Dzhioev, H. M. Gibbs, E. L. Ivchenko, G. Khitrova, V. L. Korenev, M. N. Tkachuk, and B. P. Zakharchenya, Phys. Rev. B 56, 13405 (1997).

${ }^{14}$ M. Paillard, X. Marie, P. Renucci, T. Amand, A. Jbeli, and J. M. Gérard, Phys. Rev. Lett. 86, 1634 (2001).

${ }^{15}$ T. Okuno, M. Nomura, Y. Masumoto, Y. Terai, S. Kuroda, and K. Takita, J. Phys. Soc. Jpn. 71, 3052 (2002).

${ }^{16}$ I. V. Ignaticv, I. E. Kozin, V. G. Davydov, S. V. Nair, J. S. Lee, H. W. Ren, S. Sugou, and Y. Masumoto, Phys. Rev. B 61, 15633 (2000); 63, 075316 (2001).

${ }^{17}$ R. J. Seymour and R. R. Alfano, Appl. Phys. Lett. 37, 231 (1980).

${ }^{18}$ R. R. Parsons, Can. J. Phys. 49, 1850 (1971).

${ }^{19}$ V. L. Berkovits, A. I. Ekimov and V. I. Safarov, Sov. Phys. JETP 38, 169 (1974) [Zh. Eksp. Teor. Fiz. 65, 346 (1973)]. 